\title{
TES-Based Light Detectors for the CRESST Direct Dark Matter Search
}

\author{
J. Rothe ${ }^{1}$ - G. Angloher' ${ }^{1}$ - P. Bauer' ${ }^{1}$ A. Bento ${ }^{1,9}$ - C. Bucci ${ }^{2}$. \\ L. Canonica ${ }^{2,10}$ - A. D'Addabbo ${ }^{2}$ X. Defay ${ }^{3}$ - A. Erb $^{3,11}$ - F. v. Feilitzsch ${ }^{3}$. \\ N. Ferreiro Iachellini ${ }^{1}$ - P. Gorla ${ }^{2}$ - A. Gütlein ${ }^{4,5}$ - D. Hauff ${ }^{1}$ - J. Jochum ${ }^{6}$. \\ M. Kiefer ${ }^{1}$ - H. Kluck ${ }^{4,5}$ - H. Kraus ${ }^{7}$ - J.-C. Lanfranchi ${ }^{3}$. \\ A. Langenkämper ${ }^{3}$ - J. Loebell ${ }^{6}$ - M. Mancuso ${ }^{1}$ - E. Mondragon ${ }^{3}$. \\ A. Münster ${ }^{3}$ - C. Pagliarone ${ }^{2}$ - F. Petricca ${ }^{1}$ - W. Potzel $^{3}$ - F. Pröbst ${ }^{1}$. \\ R. Puig ${ }^{4,5}$ - F. Reindl ${ }^{4,5}$ - K. Schäffner ${ }^{8}$ - J. Schieck ${ }^{4,5}$ - V. Schipperges ${ }^{6}$. \\ S. Schönert ${ }^{3}$ - W. Seidel ${ }^{1}$ - M. Stahlberg ${ }^{4,5}$. L. Stodolsky ${ }^{1}$. \\ C. Strandhagen ${ }^{6}$ - R. Strauss ${ }^{1}$ - A. Tanzke ${ }^{1}$ - H. H. Trinh Thi ${ }^{3}$. \\ C. Türkoğlu ${ }^{4,5}$ - A. Ulrich ${ }^{3}$ - I. Usherov ${ }^{6}$ - S. Wawoczny ${ }^{3}$ - M. Willers ${ }^{3}$. \\ M. Wüstrich ${ }^{1}$
}

Received: 6 November 2017 / Accepted: 30 April 2018 / Published online: 10 May 2018 (C) The Author(s) 2018

\begin{abstract}
The CRESST experiment uses cryogenic detectors based on transitionedge sensors to search for dark matter interactions. Each detector module consists of a scintillating $\mathrm{CaWO}_{4}$ crystal and a silicon-on-sapphire (SOS) light detector which operate in coincidence (phonon-light technique). The 40-mm-diameter SOS disks ( $2 \mathrm{~g}$
\end{abstract}

W. Seidel: Deceased 19/02/2017.

J. Rothe

jrothe@mpp.mpg.de

1 Max-Planck-Institute for Physics, Föhringer Ring 6, 80805 Munich, Germany

2 INFN, Laboratori Nazionali del Gran Sasso, 67010 Assergi, Italy

3 Physik-Department E15, Technische Universität München, 85747 Garching, Germany

4 Institut für Hochenergiephysik der ÖAW, 1050 Vienna, Austria

5 Atominstitut, Vienna University of Technology, 1020 Vienna, Austria

6 Eberhard-Karls-Universität Tübingen, 72076 Tübingen, Germany

7 Department of Physics, University of Oxford, Oxford OX1 3RH, UK

8 GSSI-Gran Sasso Science Institute, 67100 L'Aquila, Italy

9 LIBPhys, Departamento de Fisica, Universidade de Coimbra, 3004516 Coimbra, Portugal

10 Massachusetts Institute of Technology, Cambridge, MA 02139, USA

11 Walther-Meißner-Institut für Tieftemperaturforschung, 85748 Garching, Germany 
mass) used in the data taking campaign of CRESST-II Phase 2 (2014-2016) reached absolute baseline resolutions of $\sigma=4-7 \mathrm{eV}$. This is the best performance reported for cryogenic light detectors of this size. Newly developed silicon beaker light detectors (4 cm height, $4 \mathrm{~cm}$ diameter, $6 \mathrm{~g}$ mass), which cover a large fraction of the target crystal surface, have achieved a baseline resolution of $\sigma=5.8 \mathrm{eV}$. First results of further improved light detectors developed for the ongoing low-threshold CRESSTIII experiment are presented.

Keywords Transition-edge sensor - Cryogenic light detector · Direct dark matter search

\section{The Phonon-Light Technique in Direct Dark Matter Search}

Trying to understand the nature of dark matter is a quest that unites many branches of fundamental physics. The approach aiming to establish the particle nature of dark matter in a direct search is facing two major challenges: the smallness of the expected energy depositions induced by dark matter in the detector material, and the low rate of the expected signal. Many dark matter experiments tackle these challenges through the use of a highly sensitive primary channel for the measurement of the deposited energy, and a secondary channel for particle identification as a measure against omnipresent radiogenic backgrounds.

The cryogenic rare event search with superconducting thermometers (CRESST) experiment ([1,2] and references therein) uses tungsten thin-film TES for a highsensitivity measurement of phonons created in particle interactions in a $\mathrm{CaWO}_{4}$ target crystal at milli-Kelvin temperatures, and an SOS disk as a separate cryogenic detector to measure the scintillation light emitted from the target. Each pair of phonon and light detector is referred to as a detector module in CRESST.

As the fraction of the deposited energy emitted in scintillation light depends on the ionization density of the recoiling particle, this technique allows for event-byevent particle identification. This is a decisive advantage in dark matter searches, since radiogenic backgrounds typically result in electron-recoil events, whereas the searched-for dark matter signal is expected to produce nuclear recoils.

For analysis, each event in a CRESST dataset is assigned a light yield (LY), defined as the ratio of light energy to phonon energy for an event, normalized to 1 for $122 \mathrm{keV}$ $\gamma$ events. Electron- and $\gamma$-induced events have similar characteristics and are expected to have the same LY. $\alpha$ particles have a reduced light output (LY around 20\%). The more massive recoiling nuclei cause a further reduced scintillation output with light yields of $11 \%(\mathrm{O}), 6 \%(\mathrm{Ca}), 2 \%(\mathrm{~W})$ relative to electron-recoil events [3].

Figure 1 shows a typical plot of LY versus phonon energy obtained in a neutron calibration during CRESST-II. The two bands, which are clearly separated above a few $\mathrm{keV}$ in phonon energy, are due to neutron-induced nuclear recoils (magenta) and radiogenic electron recoils (blue), respectively. Event-by-event particle identification is thus possible down to an energy of few $\mathrm{keV}$ in this example. The performance of the light detector has an important impact on particle identification: improved light- 


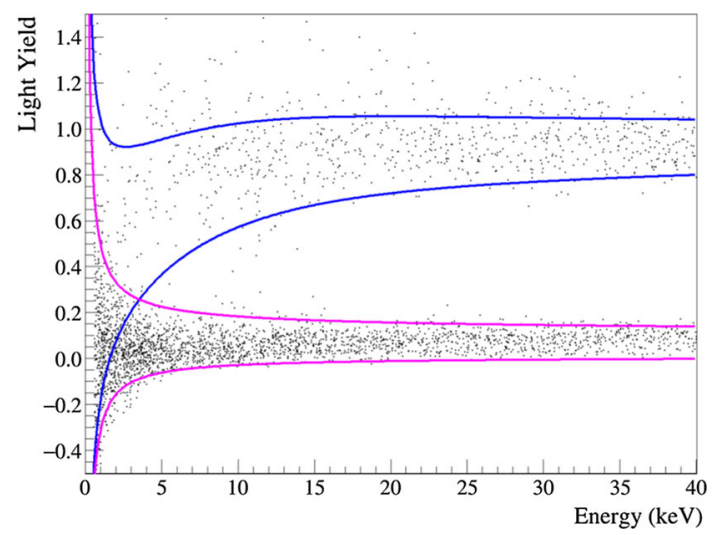

Fig. 1 Illustration of the phonon-light technique. Example of CRESST-II neutron calibration data displayed in the LY-energy plane [4]. The electron-recoil events (blue band) and nuclear-recoil events (magenta band) are separated using the information provided by the light channel. This technique allows event-by-event particle identification, decisive for background discrimination and establishing the nuclear-recoil nature of a possible signal. The blue lines denote the $90 \%$ upper and $90 \%$ lower limits of the electron-recoil band. The magenta lines show the $90 \%$ upper bound of the oxygen-recoil band and the $90 \%$ lower bound of the tungsten band. The mean of the electron-recoil band turns below one at small energies due to scintillator non-proportionality [3]. A population of events above the electron-recoil band, called excess-light events, is attributed to external radiation emitting additional light in the scintillating foil [4] (Color figure online)

detector resolution yields a more precise measurement of the LY, resulting in narrower bands. This extends the range of high-significance discrimination to lower energies.

\section{Design of the Light Channel in CRESST}

The $\mathrm{CaWO}_{4}$ crystals employed in CRESST have a scintillation yield of 20-30 photons per keV of energy deposited in an electron-recoil event. CRESST light detectors consist of an SOS disk facing the target crystal across a small gap for high light collection. Sapphire is known for its excellent properties as a cryogenic detector (high Debye temperature, therefore high sound velocities and low heat capacity). A 1- $\mu \mathrm{m}$ epitactic silicon layer allows efficient light absorption at the wavelengths emitted by $\mathrm{CaWO}_{4}$. Additionally, target crystal and light detector are surrounded by scintillating reflective foil (3M Vikuiti $\left.{ }^{\mathrm{TM}}\right)$. This further enhances light collection and leads to an additional light signal from penetrating external radiation (used for vetoing this type of background). Figure 2 summarizes the wavelength characteristics of $\mathrm{CaWO}_{4}$ emission (blue), SOS absorption (orange), and the reflectivity of the scintillating foil (green). Typically, CRESST detector modules achieve a detected light energy of $2 \%$ of the energy deposition in the target (for an electron-recoil event) [6].

Between CRESST-II and CRESST-III, the shape of the light detector has been adapted to match the target crystal geometry. In CRESST-II, cylindrical target crystals (of $40 \mathrm{~mm}$ diameter and $40 \mathrm{~mm}$ height) were equipped with SOS disks of $40 \mathrm{~mm}$ diameter and $0.4 \mathrm{~mm}$ thickness. In CRESST-III, the target crystals are 


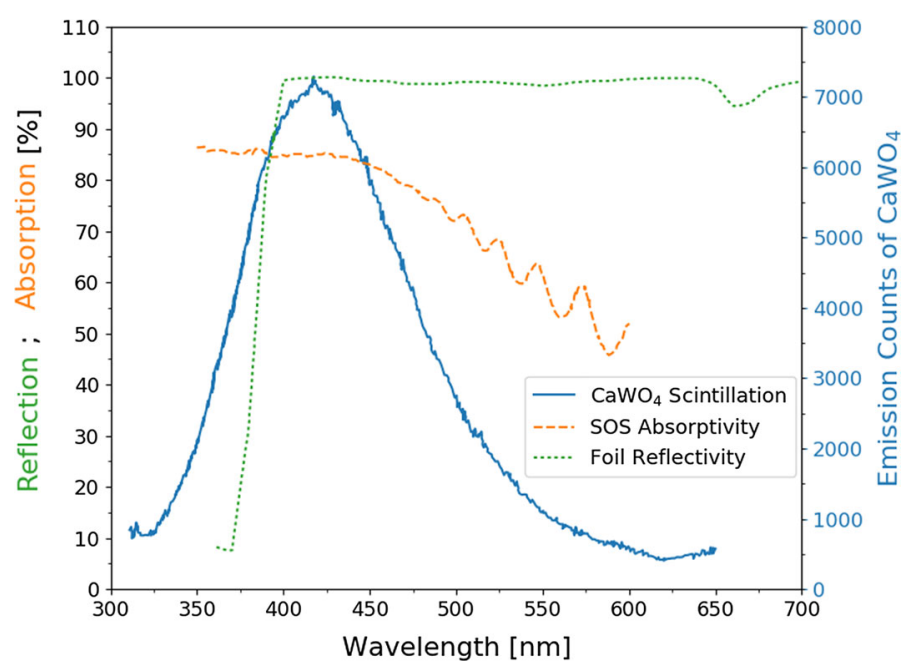

Fig. $2 \mathrm{CaWO}_{4}$ scintillation spectrum (solid blue line), wavelength-dependent reflectivity of the scintillating foil (dotted green line), and absorption of SOS wafers (dashed orange line) [5] (Color figure online)
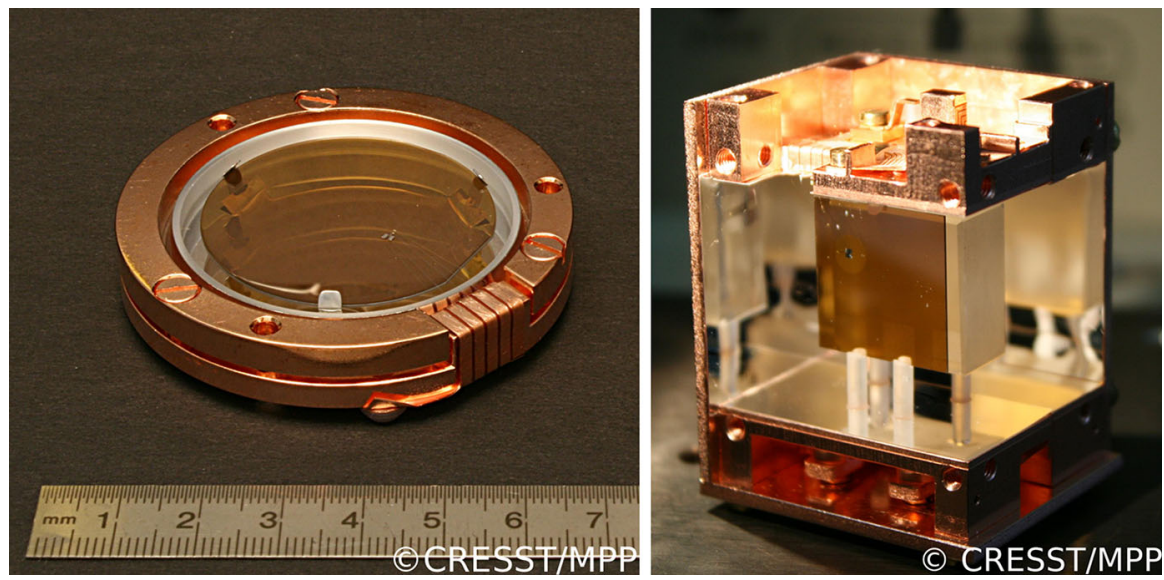

Fig. 3 Photographs of CRESST-II (left) and CRESST-III (right) light detectors (Color figure online)

$20 \times 20 \times 10 \mathrm{~mm}^{3}$ cuboids and the light detectors are $20 \times 20 \mathrm{~mm}^{2}$ square plates of $0.4 \mathrm{~mm}$ thickness. Figure 3 shows photographs of a CRESST-II light detector (left) and an opened CRESST-III module (right). The CRESST-III light detector is the vertical square plate facing the cuboid target crystal. It is held by scintillating $\mathrm{CaWO}_{4}$ sticks to avoid non-scintillating material with line-ofsight to the target crystal as a measure for vetoing any event originating in the holder $[7,8]$. 


\section{Results from CRESST-II Light Detectors}

\subsection{Characterization of the Light Detector as Part of a CRESST Detector Module}

The standard calibration technique in CRESST dark matter runs uses an external ${ }^{57}$ Co source producing $122 \mathrm{keV} \gamma$ events. The light detectors are generally strongly saturated by an absorption of a $122 \mathrm{keV} \gamma$. Due to the increasingly nonlinear detector response at high energies, this technique does not allow establishing a direct energy scale for the light detector. Instead, the coincident pulses in phonon and light channel upon absorption of a $122 \mathrm{keV} \gamma$ in the target crystal can be used to establish energy scales. In this way, a direct calibration of the phonon channel is obtained, while the light channel response is known only in electron-equivalent units $\left(\mathrm{keV}_{\mathrm{ee}}\right)$, with a conversion factor dependent on various module parameters such as scintillation yield and light collection. The energy scale of the light channel thus established can be converted to a baseline resolution of the light channel using empty baseline samples. This indirect measure of light detector resolution is most relevant for dark matter search, as it directly relates to the energy range for which particle identification is possible. The 1- $\sigma$ baseline resolution can be found from a fit to the LY bands described above. A typical value reached in a CRESST-II light detector is $250 \mathrm{eV}_{\text {ee }}$. There is a small contribution to the width of the LY band from the finite phonon channel resolution, but this is negligible compared to the light detector resolution due to the smallness of the scintillation light signal compared to the main absorber heat signal.

\subsection{Resolution of CRESST-II Light Detectors as Individual Detectors}

In order to obtain the energy scale in a light detector independent of module parameters governing the light signal generation, a direct calibration at lower energies is necessary. In CRESST-II Phase 2, several light detectors were equipped with ${ }^{55} \mathrm{Fe}$ sources which decay by electron capture and emit the characteristic $\mathrm{K}_{\alpha} / \mathrm{K}_{\beta}$ lines of $\mathrm{Mn}$ at $5.9 \mathrm{keV}$ and $6.5 \mathrm{keV}$. Figure 4 (left) shows the spectrum of the ${ }^{55} \mathrm{Fe}$ source observed in a typical light detector of CRESST-II. The baseline resolution is found from a fit of empty baseline samples. The resulting values for the baseline noise lie between $4.1 \mathrm{eV}$ and $6.7 \mathrm{eV}$. This is the best result reported for cryogenic light detectors of this size. Figure 4 (right) displays the results for light detectors equipped with ${ }^{55} \mathrm{Fe}$ sources in CRESST-II (dots: standard light detectors and crosses: beaker light detectors). As visible in Fig. 4 (left), the energy resolution is degraded at higher energies (here: $45 \mathrm{eV}$ at $6 \mathrm{keV}$ ). This is a known effect in sapphire cryogenic detectors [9].

\section{Beaker Light Detectors}

In a parallel line of development, beaker-shaped light detectors have been deployed in CRESST-II. Beaker light detectors are $40 \mathrm{~mm}$ diameter, $40 \mathrm{~mm}$ height cylindrical objects with $0.4 \mathrm{~mm}$ wall thickness milled from silicon single crystal material and optically polished. They have been paired with cylindrical target crystals of $38 \mathrm{~mm}$ 

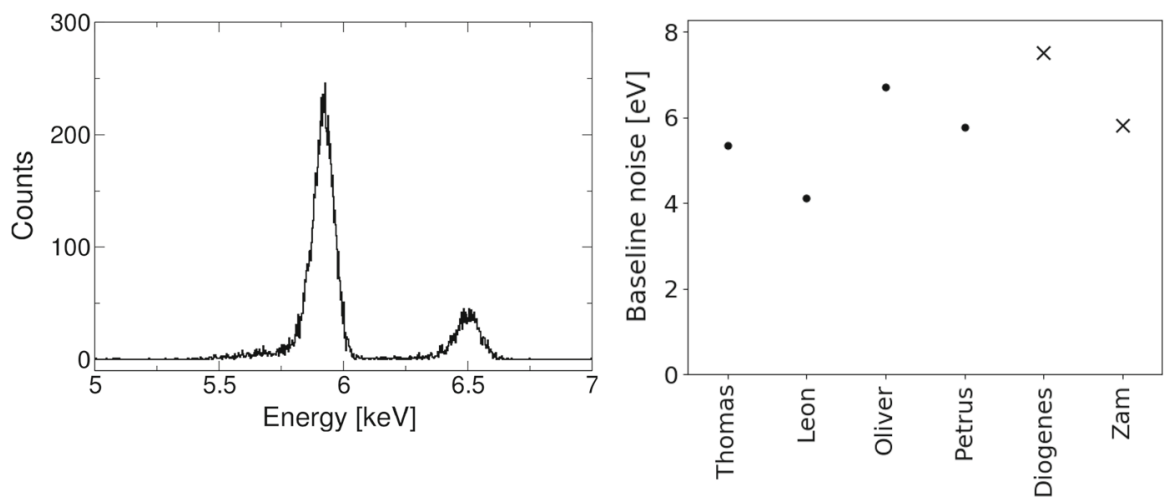

Fig. 4 Left: ${ }^{55} \mathrm{Mn} \mathrm{K}_{\alpha} / \mathrm{K}_{\beta}$ lines observed in CRESST-II light detector Leon. Right: baseline resolutions achieved by the CRESST-II light detectors equipped with ${ }^{55} \mathrm{Fe}$ sources for direct calibration. Dots: standard light detectors. Crosses: beaker light detectors
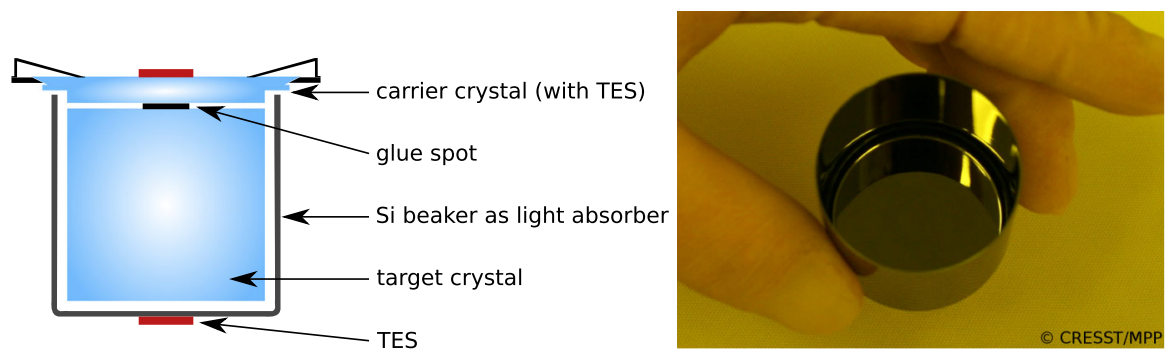

Fig. 5 Beaker light detectors. Left: CRESST beaker-module holding scheme including glued TES-carrier to complete a $4 \pi$-veto. Right: polished silicon beaker (height $40 \mathrm{~mm}$, diameter $40 \mathrm{~mm}$, thickness $0.4 \mathrm{~mm}$ ) milled from single crystal material (Color figure online)

diameter and $35 \mathrm{~mm}$ height, which were independently held to ensure a small gap between target and beaker. Excellent detector properties have been demonstrated with an achieved baseline noise of $5.8 \mathrm{eV}$ [10]. The main advantages of beaker light detectors are an enhanced light collection (up to 80\%) and an encapsulation of the target crystal. In the beaker modules, the target crystal is glued to a carrier crystal which holds the TES sensor, as shown in Fig. 5 (left). Since the target crystal has no line-ofsight to non-instrumented material, any ionizing particle that deposits energy in the target crystal also passes through the carrier and/or beaker. This results in an efficient veto for external backgrounds via light-yield and pulse-shape cuts. The same is true for surface contaminations with back-to-back event topology (either on target crystal or inner beaker surfaces). Efficient removal of such backgrounds has been shown in CRESST-II [10].

\section{Conclusion and Outlook}

Light detectors in CRESST are macroscopic absorbers equipped with a tungsten transition-edge sensor to measure temperature signals induced by the absorption of 
scintillation light. The 40-mm-diameter silicon-on-sapphire disks employed as standard light detectors in CRESST-II have reached absolute baseline resolutions of 4-7 eV $(1-\sigma)$, the best detector achieving $4.1 \mathrm{eV}$ resolution. This is the best result reported for cryogenic light detectors of this size.

Beaker light detectors milled from single-crystal silicon material have demonstrated a baseline resolution of $5.8 \mathrm{eV}$, an excellent result for a large object with a surface area of about $60 \mathrm{~cm}^{2}$. The main advantage of beaker light detectors is their greatly enhanced light collection and additional function as a veto for external radiation, as they cover a large solid angle around the target crystal.

In CRESST-III, the performance of the light channel is expected to improve in several ways. With a smaller target crystal and a more compact module, dedicated Monte Carlo studies predict a total light collection increased to $2.5 \%$ of the signal in the target. At the same time, the area of the light detector is reduced, which enhances the phonon density and transport into the thermometer. This promises an improvement in the energy resolution by a factor of 2.7. Preliminary ${ }^{57} \mathrm{Co}$-calibration data from CRESST-III show a light detector baseline noise of $80 \mathrm{eV}_{\mathrm{ee}}$, improved by a factor of three compared to CRESST-II performance, consistent with the expectations. With the light collection predicted by the Monte Carlo studies, this translates into a direct baseline resolution in the range of $2 \mathrm{eV}$. Though not crucial for the application in dark matter search, it is interesting to note that the macroscopic light detectors used in CRESST approach calorimetric single-photon resolution. Further study and direct calibration measurements are needed to confirm the resolution of CRESST-III light detectors.

Acknowledgements Open access funding provided by Max Planck Society.

Open Access This article is distributed under the terms of the Creative Commons Attribution 4.0 International License (http://creativecommons.org/licenses/by/4.0/), which permits unrestricted use, distribution, and reproduction in any medium, provided you give appropriate credit to the original author(s) and the source, provide a link to the Creative Commons license, and indicate if changes were made.

\section{References}

1. F. Petricca et al. (CRESST Collaboration), J. Phys. Conf. Ser. arXiv:1711.07692

2. G. Angloher et al. (CRESST Collaboration), Eur. Phys. J. C 76, 25 (2016). https://doi.org/10.1140/ epjc/s10052-016-3877-3

3. R. Strauss et al. (CRESST Collaboration), Eur. Phys. J. C 74, 2957 (2014). https://doi.org/10.1140/ epjc/s10052-014-2957-5

4. F. Reindl, Dissertation, TU München, mediatum.ub.tum.de?id=1294132 (2016)

5. P. Huff, Dissertation, TU München (2010), mediatum.ub.tum.de?id=977540

6. M. Kiefer (CRESST Collaboration). Nucl. Instrum. Methods Phys. Res. A 821, 0168-9002 (2016). https://doi.org/10.1016/j.nima.2016.03.035

7. R. Strauss et al. (CRESST Collaboration), Eur. Phys. J. C 75, 352 (2015). https://doi.org/10.1140/epjc/ s10052-015-3572-9

8. R. Strauss et al. (CRESST Collaboration). Nucl. Instrum. Methods Phys. Res. A 845, 0168-9002 (2017). https://doi.org/10.1016/j.nima.2016.06.060

9. M. Sisti et al., Nucl. Instrum. Methods A 466, 499-508 (2001). https://doi.org/10.1016/S01689002(01)00801-4

10. G. Angloher et al. (CRESST Collaboration). arXiv:1708.01581 (2017) 